\title{
Anfíbios anuros em uma área de Mata Atlântica da Serra do Tabuleiro, Santa Catarina
}

\author{
Milena Wachlevski ${ }^{1 *}$ \\ Luciana Kreutz Erdtmann ${ }^{2}$ \\ Paulo Christiano de Anchietta Garcia ${ }^{3}$ \\ ${ }^{1}$ Laboratório de Gestão e Conservação da Fauna Silvestre, Departamento de Ciências Animais \\ Universidade Federal Rural do Semi-árido \\ Avenida Francisco Mota, 572, CEP 59625-900, Mossoró - RN, Brasil \\ ${ }^{2}$ PPG em Ecologia, Instituto Nacional de Pesquisas da Amazônia, Manaus - AM, Brasil \\ ${ }^{3}$ Departamento de Zoologia, Instituto de Ciências Biológica \\ Universidade Federal de Minas Gerais, Belo Horizonte - MG, Brasil \\ * Autor para correspondência \\ milenawm@yahoo.com
}

Submetido em 18/09/2013

Aceito para publicação em 17/03/2014

\section{Resumo}

A Mata Atlântica é uma área prioritária para a conservação de anfíbios, com algumas regiões que ainda apresentam lacunas de conhecimento. Analisamos a composição e riqueza das espécies de anuros em área de floresta ombrófila densa da Serra do Tabuleiro, a variação sazonal da riqueza e a atividade diária dos machos em turnos de vocalização. Amostramos os anuros em duas lagoas permanentes e em uma trilha no interior da floresta durante 14 meses. Registramos 32 espécies de anuros, das quais Aplastodiscus cochranae, A. ehrhardti e Hypsiboas poaju estão na lista de espécies ameaçadas do estado. O maior número de espécies esteve associado à primavera e ao verão. As espécies de anuros mais frequentes foram H. bischoffi, Adenomera araucaria e Physalaemus nanus, registradas durante todo o período estudado. A atividade diária dos machos esteve concentrada entre 20:00 e 24:00, mas algumas espécies continuaram vocalizando durante a madrugada, indicando que a atividade vocal pode diferir entre as espécies submetidas às mesmas condições climáticas. A diversidade de anuros registrada em nosso estudo foi alta, incluindo espécies ameaçadas e espécies com pouco conhecimento biológico, reforçando a relevância da Serra do Tabuleiro como área prioritária para a conservação da Mata Atlântica.

Palavras-chave: Anfíbios; Atividade diária; Riqueza de espécies; Sazonalidade; Sul do Brasil

\section{Abstract}

Anuran amphibians in an Atlantic Forest area at Serra do Tabuleiro, Santa Catarina, Brazil. The Atlantic Forest is a priority area for the conservation of amphibians, with some regions already showing knowledge gaps. We analyzed the composition and richness of anuran species in an area of dense ombrophilous forest at Serra do Tabuleiro, the seasonal richness variation, and the daily activity of males during vocalization shifts. We collected samples of anurans from two permanent ponds and from a track within the forest for 14 months. We recorded 32 anuran species, among which Aplastodiscus cochranae, A. ehrhardti, and Hypsiboas poaju are 
included in the list of endangered species in the state. The highest number of species was associated to spring and summer. The most frequent anuran species were H. bischoffi, Adenomera araucaria, and Physalaemus nanus, registered throughout the study period. The daily activity of males was concentrated between $8 \mathrm{p} . \mathrm{m}$. and 12 p.m., but some species keep vocalizing overnight, indicating that vocal activity can differ among species undergoing the same weather conditions. The diversity of anurans recorded in our study was high, including endangered species and species with poor biological knowledge, reinforcing the relevance of Serra do Tabuleiro as a priority area for preserving the Atlantic Forest.

Key words: Amphibia; Daily activity; Seasonality; Southern Brazil; Species richness

\section{Introdução}

A Mata Atlântica abriga uma das maiores diversidades de anfíbios do mundo, com espécies endêmicas e com o maior número de espécies ameaçadas do Brasil (SUBIRÁ et al., 2012; HADDAD et al., 2013). O estado de Santa Catarina está inteiramente inserido no bioma Mata Atlântica, mas atualmente possui apenas $21 \%$ de remanescentes expostos à crescente pressão de desmatamento nos últimos anos (FUNDAÇÃO SOS MATA ATLÂNTICA, 2010).

Considerando que aproximadamente um quarto das espécies de anuros da Mata Atlântica ocorre em Santa Catarina (LUCAS, 2008; HADDAD et al., 2013), que existem espécies endêmicas e ameaçadas no estado (e.g. Hypsiboas poaju GARCIA et al., 2008; CONSEMA/ SC resolução 002 de 06 de dezembro de 2011) e que há inúmeras lacunas de conhecimento acerca da diversidade de anfíbios (LUCAS, 2008), a compreensão da estrutura das comunidades de anfíbios é relevante para promover a conservação deste grupo, principalmente em regiões de grande riqueza e pouca informação disponível.

A Serra do Tabuleiro possui um dos maiores remanescentes de Mata Atlântica do sul do Brasil, está localizada no leste catarinense e é uma das áreas prioritárias para a conservação de anfíbios na Mata Atlântica (MMA, 2000). O conhecimento sobre os anuros da Serra do Tabuleiro inclui uma lista de espécies para uma área costeira de restinga incluída no Parque Estadual da Serra do Tabuleiro (WACHLEVSKI; ROCHA, 2010), registros de distribuição geográfica (GARCIA; VINCIPROVA, 1998), espécimes depositados em museus (KUNZ et al., 2007; LUCAS; GARCIA, 2011) e trabalhos taxonômicos como revisões e descrições de novas espécies (e.g. HEYER, 1985; GARCIA et al., 2001; 2008; NUNES et al., 2012).
A dinâmica sazonal na atividade reprodutiva das espécies de anuros é influenciada por uma interação complexa de variáveis ambientais bióticas e abióticas como temperatura, fotoperíodo e pluviosidade, além dos ciclos endógenos da físiologia dos organismos (DUELLMAN; TRUEB, 1994). Os meses do ano são frequentemente utilizados como uma unidade de medida sazonal da atividade dos anuros, refletindo a maior parte da amplitude das variáveis que influenciam suas atividades ao longo do ano (CANAVERO et al., 2008). $A$ atividade dos anuros parece seguir primariamente duas escalas temporais, uma sazonal na qual os indivíduos estão ativos em ciclos anuais e outra que corresponde às respostas curtas, como a oscilação na atividade diária, nas quais a temperatura e/ou a pluviosidade influenciam a atividade de vocalização dos anuros em um dia ou semana (CARDOSO; MARTINS, 1987; CANAVERO et al., 2008).

Os objetivos deste estudo foram estimar a riqueza, listar a composição e verificar a distribuição temporal das espécies de anuros em uma área de Mata Atlântica da Serra do Tabuleiro, no estado de Santa Catarina. Também avaliamos o período de atividade dos anuros em turnos de vocalização em lagoas em áreas abertas e em uma trilha no interior da floresta.

\section{Material e Métodos}

\section{Área de estudo}

O estudo foi realizado em uma área de Mata Atlântica com 138 ha $\left(27^{\circ} 43^{\prime}\right.$ S, $\left.48^{\circ} 49^{\prime} \mathrm{W}\right)$ pertencente ao Hotel Plaza Caldas da Imperatriz (HPCI), localizado no noroeste da Serra do Tabuleiro, município de Santo Amaro da Imperatriz, estado de Santa Catarina, Brasil. 
A vegetação é caracterizada como Floresta Ombrófila Densa Submontana (KLEIN, 1981), com altitudes que variam de 220 a $490 \mathrm{~m}$. O clima da região é classificado como temperado, sem estação seca e com verões quentes (Cfa) (PEEL et al., 2007). A temperatura média mensal varia entre 15 e $25^{\circ} \mathrm{C}$, com uma precipitação média anual de $1.600 \mathrm{~mm}$, sendo os meses de janeiro a abril os mais chuvosos (dados fornecidos pelo Centro de Informações de Recursos Ambientais e de Hidrometeorologia de Santa Catarina/Empresa de Pesquisa Agropecuária e Extensão Rural de Santa Catarina - CIRAM/EPAGRI 1990-2000 - http://ciram.epagri.sc.gov.br).

Amostramos os anuros em duas lagoas permanentes, uma delas (L1) possuía uma área superficial aproximada de $1.000 \mathrm{~m}^{2}$, situada a $240 \mathrm{~m}$ de altitude. Sua margem era composta por vegetação arbustiva com papiros (Cyperus papirus), vegetação nativa arbustiva (e.g. Melastomataceae), com fundo lodoso e na região central, a profundidade da água era superior a dois metros. No entorno de L1 existiam pequenas áreas alagadas que também foram consideradas como parte desse ambiente. Já a segunda lagoa permanente (L2) possuía uma área superficial de $1.480 \mathrm{~m}^{2}$, situada a $420 \mathrm{~m}$ de altitude e sua margem era composta por arbustos e arvoretas nativas, fundo lodoso e profundidade superior a dois metros. A vegetação a qual a trilha no interior da floresta (T) estava inserida era composta por vegetação nativa em estágio secundário de regeneração, possuía extensão de um quilometro e altitude variando de 420 a 490 m. A trilha foi amostrada de maneira a abranger a maior heterogeneidade de ambientes que pudesse abrigar as espécies de anuros presentes na floresta, como ambientes de folhiço, bromélias, cinco riachos, cinco poças temporárias e duas poças permanentes de fundo lodoso que estavam dispostos próximos ou interceptados pela trilha.

$\mathrm{O}$ esforço de amostragem ao longo da trilha foi distribuído equitativamente com uma amplitude de procura de dois metros para cada lado. O início da trilha era próximo da L2 cerca de $50 \mathrm{~m}$ e ambos estavam a cerca de 970 m de distância da L1.

\section{Coleta e análise de dados}

Fizemos visitas mensais na área de estudo de fevereiro de 2001 a março de 2002. Em cada mês os anuros foram amostrados durante duas noites, entre $18 \mathrm{~h} 00 \mathrm{~min}$ e $01 \mathrm{~h} 00 \mathrm{~min}$ e duas manhãs, entre $08 \mathrm{~h} 00 \mathrm{~min}$ e $11 \mathrm{~h} 00 \mathrm{~min}$, por meio de procura visual revirando galhos, folhas, folhiço e pequenas poças (CRUMP; SCOTT JR, 1994) na trilha T e por busca em sítios reprodutivos (SCOTT JR; WOODWARD, 1994) nas duas lagoas permanentes L1 e L2. O mesmo método de amostragem utilizado nas lagoas permitiu a comparação direta na variação da riqueza e composição das espécies nesses sítios reprodutivos. Entre 1999 e 2001, fizemos buscas ocasionais na área de estudo e esses dados foram contabilizados para a riqueza total e composição de espécies, mas não entraram nas análises com relação à sazonalidade.

As buscas por anuros foram realizadas por dois observadores, totalizando um esforço de 280 horaspessoa para as amostragens noturnas e 168 horas-pessoa de esforço amostral diurno. O início das buscas era alternado entre a trilha e as lagoas para reduzir o efeito do horário sobre os ambientes amostrados.

Avaliamos se o esforço amostral foi suficiente para amostrar a comunidade de anfíbios anuros local construindo uma curva de acumulação de espécies, verificando se esta curva atingiu uma assíntota e estimamos a riqueza de espécies por meio do estimador Jackknife 1 com mil aleatorizações, utilizando dados de presença e ausência de cada espécie por cada dia de procura desde 1999. Utilizamos o programa EstimateS 8.2 para construir as curvas de riqueza de espécies, utilizando aleatorizações sobre os dados originais (SOBS) (COLWELL, 2009).

Para analisar a distribuição temporal na riqueza de espécies, fizemos uma análise de estatística circular (ZAR, 1999), utilizando o programa ORIANA 2.0 (KOVACH, 2004). Limitamos o período de análise para um ciclo de um ano, considerando os dados de riqueza de abril de 2001 a março de 2002, excluindo os meses de fevereiro e março de 2001. Os 12 meses amostrados (abril de 2001 a março de 2002) foram convertidos pelo programa em ângulos com intervalos de $30^{\circ}$ e o número de espécies presentes em cada mês correspondeu às frequências de cada ângulo observado (modificado de PRADO et al., 2005). Estimamos o ângulo médio $(\alpha)$ e o desvio padrão circular $(S D)$ relativo ao $\alpha$. O ângulo médio é a média de uma amostra de $n$ ângulos, 
representando cada espécie registrada nos intervalos de $30^{\circ}$, correspondendo a cada mês como total de ângulos do círculo de $360^{\circ}$, ou seja, os 12 meses. O ângulo médio mais próximo do valor de $360^{\circ}$ significa que os dados estão dispersos ao longo do círculo, enquanto que, mais próximo de $0^{\circ}$, os dados estão mais concentrados em uma ou algumas direções (ZAR, 1999). Também calculamos o comprimento do vetor médio ( $r$ ), que é uma medida de concentração dos dados de maior número de espécies, ângulos, no entorno do círculo de 12 meses, podendo variar de zero a um. Se o valor do comprimento for próximo de zero, significa que a riqueza de espécies foi dispersa ao longo dos meses e quanto mais próximo de um, o número de espécies seria maior em uma mesma direção, ou seja, em um mesmo mês (ZAR, 1999).

Utilizamos o teste de uniformidade de Rayleigh para calcular a probabilidade dos dados de riqueza de espécies de anuros serem distribuídos uniformemente ao longo dos meses $(P>0,05)$ (ZAR, 1999). Um resultado significativo para o teste de Rayleigh indicaria que a riqueza de espécies não foi distribuída uniformemente ao longo dos meses amostrados durante este estudo.

Analisamos dois turnos diários de vocalização em janeiro de 2002 com início às 06h00min horas de um dia e término às $05 \mathrm{~h} 00 \mathrm{~min}$ horas do dia seguinte $\mathrm{e}$ desconsideramos o horário de verão, para avaliar qual o período do dia em que houve um maior número de espécies de anuros em atividade de vocalização e qual o período de maior abundância estimada. Contabilizamos o número de machos de cada espécie que estava vocalizando a cada hora nos locais: L1 (24 de janeiro de 2002), em L2 e em T (ambos no dia 15 de janeiro de 2002). Usamos uma medida conservativa para estimar o número de machos vocalizando, categorizando de um a cinco indivíduos, de seis a dez indivíduos e mais de dez indivíduos. Durante os turnos de vocalização, a temperatura do ar foi registrada a cada hora.

A nomenclatura científica seguiu Frost (2013). Os espécimes testemunhos foram depositados no Museu de História Natural Capão da Imbuia (MHNCI 5000-125), Curitiba, Paraná.

\section{Resultados}

Registramos 32 espécies de anfíbios anuros distribuídas em oito famílias (Tabela 1). Destas, 28 espécies foram registradas nas amostragens entre 2001 e 2002. A riqueza estimada de espécies $(32,96 \pm 0,97$ espécies) (Jackknife) foi semelhante à riqueza observada (Figura 1), indicando que amostramos a maior parte das espécies que ocorrem no local.

FIGURA 1: Curva ajustada de acumulação de espécies (ロ) e da estimativa de riqueza pelo método de Jackknife 1 ( $\square$ ) das espécies de anfíbios anuros registradas em uma área de Floresta Ombrófila Densa Submontana, Santo Amaro da Imperatriz, estado de Santa Catarina. As linhas verticais acima e abaixo dos pontos correspondem ao desvio padrão das estimativas.

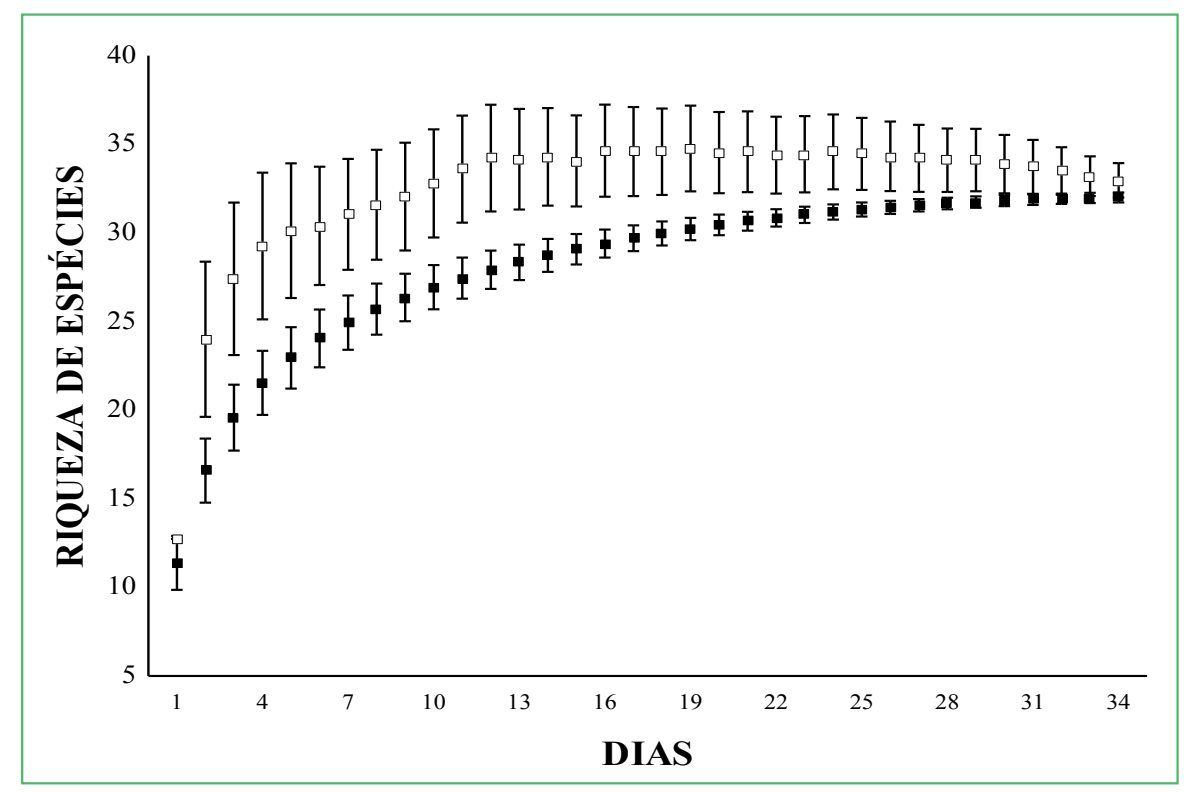


TABELA 1: Espécies de anfíbios anuros registradas em uma área de Floresta Ombrófila Densa Submontana em Santo Amaro da Imperatriz, estado de Santa Catarina, durante 1999 a 2002 em duas lagoas artificiais (L1 e L2) e em uma trilha no interior da floresta $(\mathrm{T})$.

\begin{tabular}{|c|c|c|c|}
\hline Família/espécie & L1 & $\mathbf{L 2}$ & $\mathbf{T}$ \\
\hline \multicolumn{4}{|l|}{ Brachycephalidae } \\
\hline Ischnocnema henselii (Peters, 1970) & - & - & $\mathrm{X}$ \\
\hline \multicolumn{4}{|l|}{ Bufonidae } \\
\hline Dendrophryniscus berthalutzae Izecksohn, 1994 & - & - & $\mathrm{X}$ \\
\hline Rhinella abei (Baldissera, Caramaschi \& Haddad, 2004) & $\mathrm{X}$ & $\mathrm{X}$ & $\mathrm{X}$ \\
\hline Rhinella icterica (Spix, 1824) & $\mathrm{X}$ & $\mathrm{X}$ & - \\
\hline \multicolumn{4}{|l|}{ Craugastoridae } \\
\hline Haddadus binotatus (Spix, 1824) & - & - & $\mathrm{X}$ \\
\hline \multicolumn{4}{|l|}{ Hemiphractidae } \\
\hline Fritziana aff. fissilis & - & - & $\mathrm{X}$ \\
\hline \multicolumn{4}{|l|}{ Hylidae } \\
\hline Aplastodiscus cochranae (Mertens, 1952) & - & - & $\mathrm{X}$ \\
\hline Aplastodiscus ehrhardti (Müller, 1924) & - & - & $\mathrm{X}$ \\
\hline Bokermannohyla hylax (Heyer, 1985) & - & - & $\mathrm{X}$ \\
\hline Dendropsophus microps (Peters, 1872) & - & - & $\mathrm{X}$ \\
\hline Dendropsophus minutus (Peters, 1872) & $\mathrm{X}$ & $\mathrm{X}$ & - \\
\hline Dendropsophus nahdereri (B. Lutz \& Bokermann, 1963) & - & - & $\mathrm{X}$ \\
\hline Hypsiboas bischoffi (Boulenger, 1887) & $\mathrm{X}$ & $\mathrm{X}$ & $\mathrm{X}$ \\
\hline Hypsiboas faber (Wied-Beuwied, 1821) & $\mathrm{X}$ & $\mathrm{X}$ & - \\
\hline Hypsiboas роајu Garcia, Peixoto \& Haddad, 2008* & - & - & $\mathrm{X}$ \\
\hline Phyllomedusa distincta Lutz, 1950 & $\mathrm{X}$ & $\mathrm{X}$ & - \\
\hline Scinax catharinae (Boulenger, 1888)* & - & - & $\mathrm{X}$ \\
\hline Scinax perereca Pombal, Haddad \& Kasahara, 1995 & $\mathrm{X}$ & $\mathrm{X}$ & $\mathrm{X}$ \\
\hline Scinax rizibilis (Bokermann, 1964) & - & - & $\mathrm{X}$ \\
\hline Scinax tymbamirim Nunes, Kwet \& Pombal Jr. 2012 & $\mathrm{X}$ & - & - \\
\hline Trachycephalus mesophaeus (Hensel, 1867) & - & - & $\mathrm{X}$ \\
\hline \multicolumn{4}{|l|}{ Hylodidae } \\
\hline Hylodes aff. perplicatus & - & - & $\mathrm{X}$ \\
\hline \multicolumn{4}{|l|}{ Leptodactylidae } \\
\hline Adenomera araucaria Kwet \& Ângulo, 2002 & - & $\mathrm{X}$ & $\mathrm{X}$ \\
\hline Adenomera engelsi Kwet, Steiner \& Zillikens, 2009 & $\mathrm{X}$ & $\mathrm{X}$ & $\mathrm{X}$ \\
\hline Leptodactylus gracilis (Duméril \& Bibron, 1840) & $\mathrm{X}$ & - & $\mathrm{X}$ \\
\hline Leptodactylus latrans (Steffen, 1815)* & $\mathrm{X}$ & - & - \\
\hline Physalaemus cuvieri Fitzinger, 1826 & $\mathrm{X}$ & $\mathrm{X}$ & - \\
\hline Physalaemus lateristriga (Steindachner, 1864) & - & - & $\mathrm{X}$ \\
\hline Physalaemus nanus (Boulenger, 1888) & $\mathrm{X}$ & - & $\mathrm{X}$ \\
\hline Scythrophrys aff. sawayae* & - & - & $\mathrm{X}$ \\
\hline \multicolumn{4}{|l|}{ Odontophrynidae } \\
\hline Proceratophrys boiei (Wied-Beuwied, 1824) & - & - & $\mathrm{X}$ \\
\hline Proceratophrys subguttata Izecksohn, Cruz e Peixoto, 1999 & - & - & $\mathrm{X}$ \\
\hline TOTAL DE ESPÉCIES & 13 & 10 & 25 \\
\hline
\end{tabular}

* Espécies que ocorreram na área de estudo, mas não foram registradas entre fevereiro de 2001 e março de 2002. 
A riqueza de espécies de anuros não esteve distribuída uniformemente ao longo do ano $(Z=18,66$; $P<0,001$ ) (Figura 2), com um maior número de espécies nos meses correspondentes às estações de primavera e verão, com 18 a 25 espécies de outubro a fevereiro, enquanto no outono e no inverno registramos de seis a 15 espécies $\left(\alpha=236,35^{\circ}, D P=84,49^{\circ}, r=0,33\right)$ (Figuras 2 e 3).

Hypsiboas bischoffi e Adenomera araucaria foram as espécies mais frequentes neste estudo, sendo registradas em todos os meses amostrados, seguidas por Physalaemus nanus, presentes em 93\% os meses amostrados. A espécie menos frequente foi Proceratophrys subguttata, encontrada apenas em junho de 2001, com 7,1\% da ocorrência total (Figura 3). Oito espécies $(28,5 \%)$ ocorreram apenas na primavera e verão, uma espécie, $P$. subguttata, ocorreu apenas no outono e nenhuma espécie foi registrada exclusivamente no inverno.

Durante o turno de vocalização, 12 espécies estiveram em atividade na L1, seis espécies na L2 e 14 espécies no interior da floresta $(\mathrm{T})$. $\mathrm{O}$ maior número de espécies vocalizando ocorreu logo após anoitecer, contabilizados às $20 \mathrm{~h} 00 \mathrm{~min}, 83 \%$ das espécies em atividade vocal na L1, 67\% das espécies na L2 e 64\% dos anuros na floresta (Figura 4). Hylodes aff. perplicatus e Adenomera araucaria vocalizaram continuamente durante o dia, sendo que a primeira espécie apresentou atividade de vocalização estritamente diurna, encerrando-a logo após o crepúsculo. Nas lagoas, Hypsiboas faber e Physalaemus nanus vocalizaram esporadicamente durante o dia, mas concentraram a atividade de vocalização durante a noite (Figura 4).

FIGURA 2: Diagrama de rosa da análise circular para a riqueza de espécies de anfíbios anuros entre abril de $2001\left(0^{\circ}\right)$ a março de 2002 $\left(330^{\circ}\right)$, em uma área de Floresta Ombrófila Densa Submontana, município de Santo Amaro da Imperatriz, estado de Santa Catarina. O comprimento médio do vetor indicado pela seta $(r=0,33)$ é uma medida de concentração da riqueza de espécies de anuros ao longo dos meses.

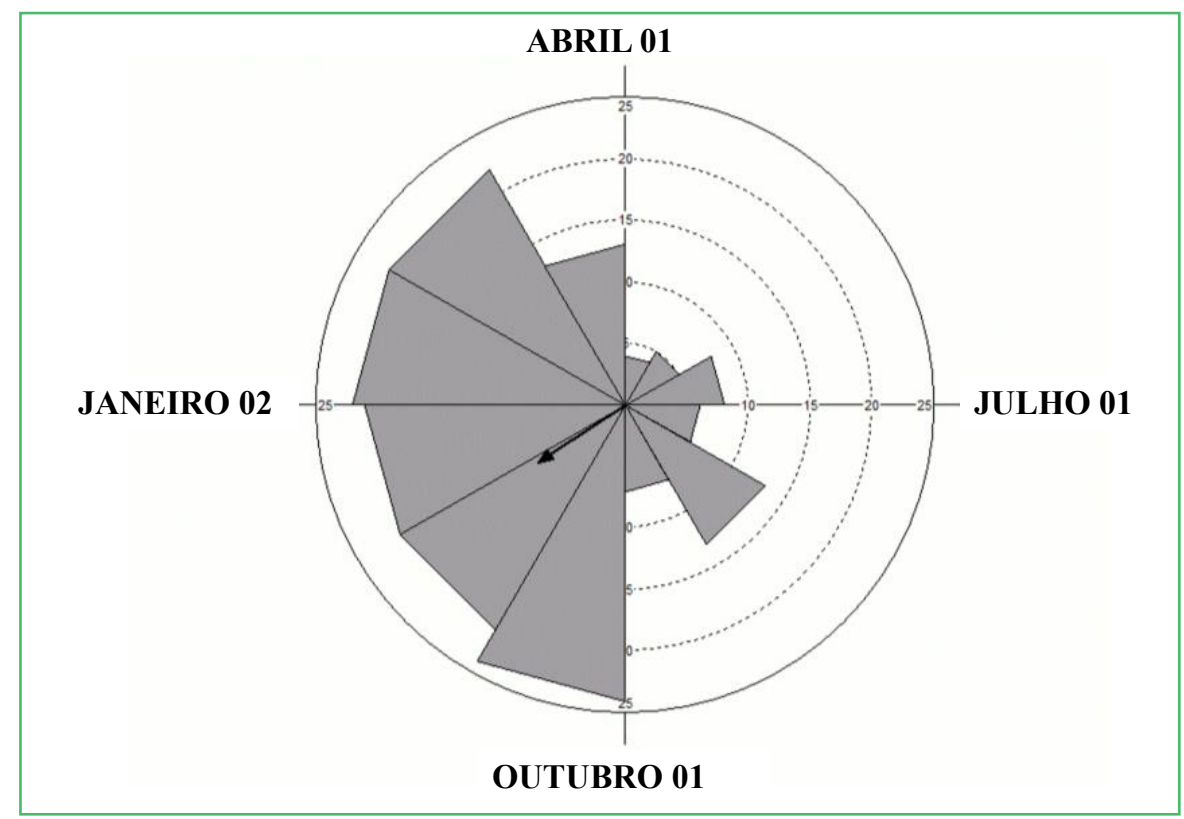


FIGURA 3: Ocorrência mensal das espécies de anfíbios anuros em uma área de Foresta Ombrófila Densa Submontana, em Santo Amaro da Imperatriz, estado de Santa Catarina, durante fevereiro de 2001 a março de 2002.

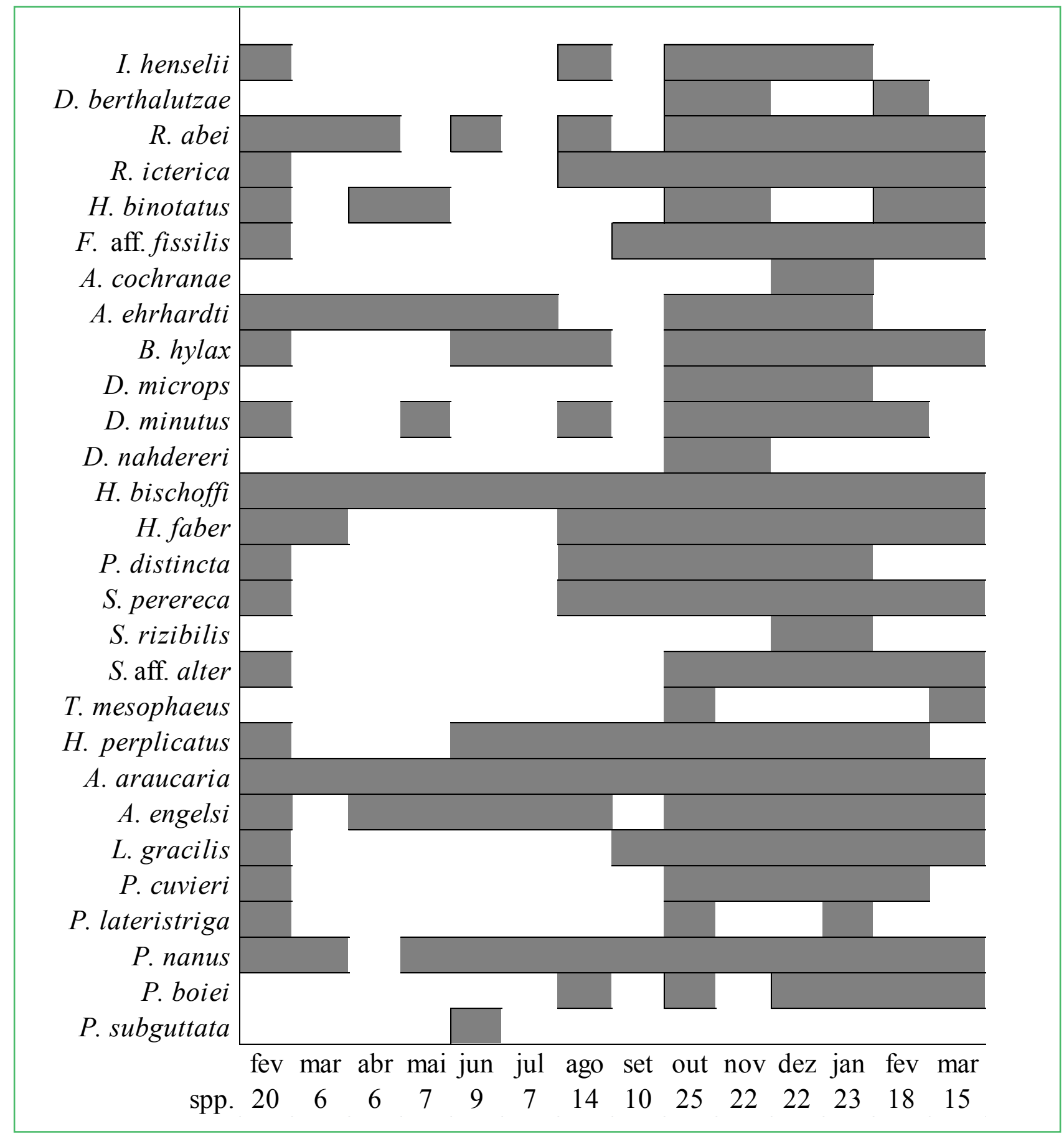


FIGURA 4: Turnos de vocalização das espécies de anuros registradas na Lagoa 1 (L1) em 24 de janeiro de 2002; na Lagoa 2 (L2) e na trilha no interior da floresta (T), ambas amostradas no dia 15 de janeiro de 2002, em uma área de Floresta Ombrófila Densa Submontana, em Santo Amaro da Imperatriz, estado de Santa Catarina. Os turnos de vocalização foram realizados a cada hora das $06 \mathrm{~h} 00 \mathrm{~min}$ as $05 \mathrm{~h} 00 \mathrm{~min}$, a hora do ocaso está em negrito. Os dados de temperatura do ar foram registrados em graus Celsius. O número de espécies vocalizando por hora está no topo de cada gráfico e as categorias de abundância de machos vocalizando foram: cinza claro - menos que cinco indivíduos; cinza escuro - de cinco a dez indivíduos e; preto - mais de dez indivíduos.

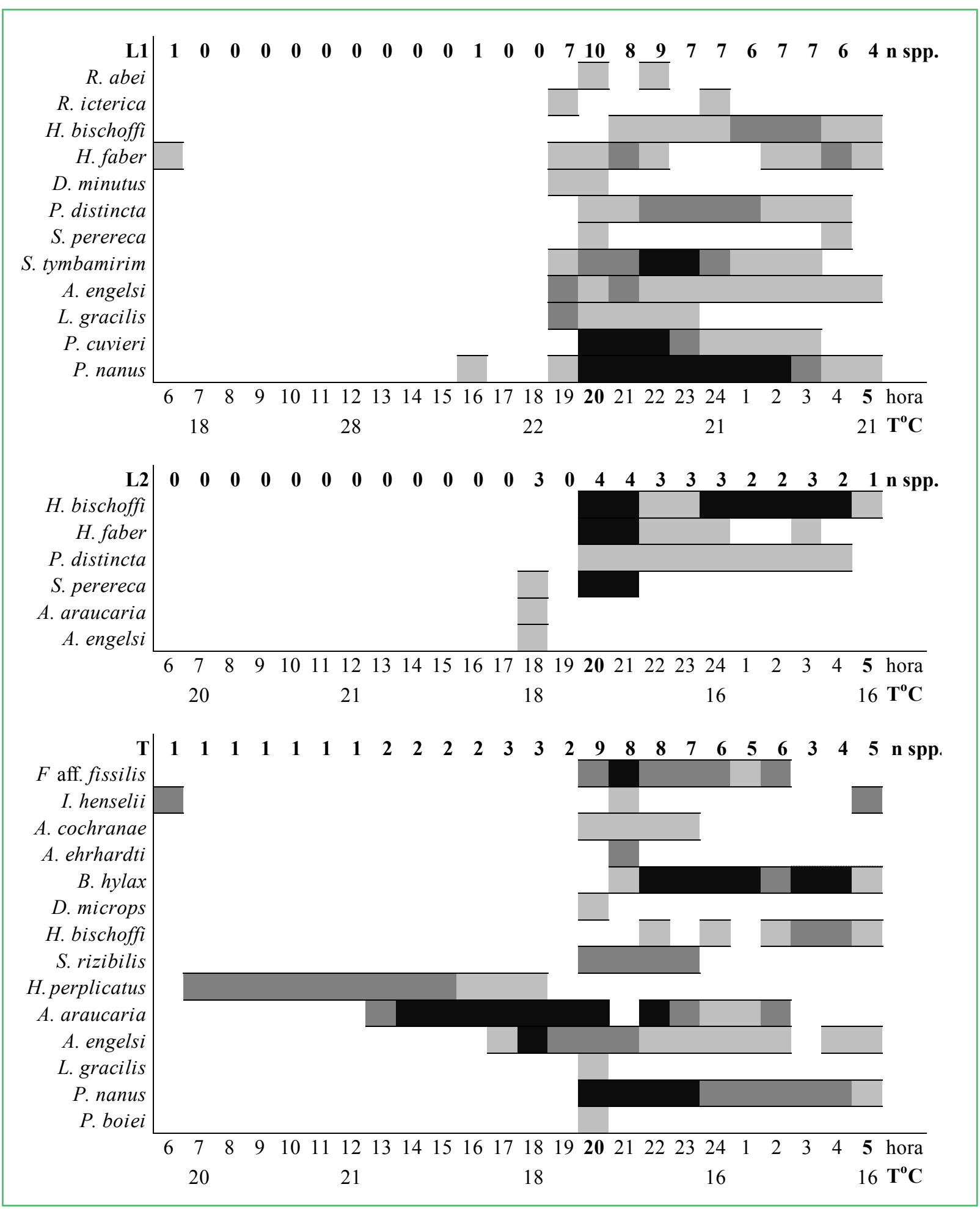


A riqueza de espécies e a abundância relativa dos anuros tenderam a ser maior logo após o ocaso, que ocorreu as $20 \mathrm{~h} 00 \mathrm{~min}$, até cerca das $23 \mathrm{~h} 00 \mathrm{~min}$, mas muitas espécies se mantiveram em atividade de vocalização durante a madrugada, $67 \%$ das espécies na L1, 50\% na L2 e 43\% na floresta. A maior abundância dos machos de $H$. bischoffi ocorreu na segunda metade da noite (entre 24 e $4 \mathrm{~h} 00 \mathrm{~min}$ ) nos três ambientes (Figura 4). Physalaemus nanus e Bokermannohyla hylax também estiveram ativos durante toda a noite.

\section{Discussão}

A riqueza de espécies do HPCI correspondeu a cerca de 30\% das espécies de anuros conhecidas para Santa Catarina (LUCAS, 2008) e seis por cento das espécies de anfíbios da Mata Atlântica (HADDAD et al., 2013), indicando a alta diversidade regional na Serra do Tabuleiro. O registro de algumas espécies durante a maior parte dos meses amostrados pode estar relacionado a espécies comuns na região, como Hypsiboas bischoffi (MARCELINO et al., 2009), espécies que possuem atividade reprodutiva prolongada e/ou ficam ativas após eventos de chuva, como Adenomera araucaria e Physalaemus nanus. Por outro lado, espécies que se reproduzem em poças temporárias, como Scinax rizibilis e Physalaemus lateristriga, ou que possuem reprodução explosiva, como Trachycephalus mesophaeus (KWET et al., 2010), tendem a ocorrer em períodos mais quentes e chuvosos, quando se formam os sítios reprodutivos no interior ou na borda da floresta.

Destacamos também a presença de três espécies classificadas como vulneráveis na lista de espécies ameaçadas de Santa Catarina (CONSEMA/SC resolução 002/de 06 de dezembro de 2011): Aplastodiscus cochranae, A. ehrhardti e Hypsiboas poaju. Esta última também foi incluída na lista nacional de espécies ameaçadas, na categoria quase ameaçada (SUBIRÁ et al., 2012). O registro dessas espécies na área pode subsidiar futuras ações e estudos para a conservação de suas populações.

A riqueza e a composição de espécies de anuros variaram sazonalmente com uma maior diversidade registrada nos meses de outubro a fevereiro, que correspondem às estações de primavera e verão. A atividade dos anuros é dependente de fatores ambientais como pluviosidade, umidade relativa do ar e temperatura (DUELLMAN; TRUEB, 1994). Na Floresta Ombrófila Densa subtropical, a variação sazonal tende a ser mais dependente da associação entre a temperatura e maiores índices pluviométricos do que somente à alta pluviosidade, já que a variação de temperatura é maior do que em florestas tropicais. A maior riqueza de anuros ocorreu na primavera e verão também em São José dos Pinhais, Paraná e Santa Maria, no Rio Grande do Sul (CONTE; MACHADO, 2005; SANTOS et al., 2008). Determinar todos os fatores que contribuem para a ocorrência sazonal dos anuros em uma comunidade é complexo, mas as estações do ano podem fornecer bons indicativos de sazonalidade para a comparação entre comunidades de anfíbios (CANAVERO et al., 2008), como ocorreu na comunidade amostrada por nós.

Os machos estiveram mais ativos vocalmente durante a noite, o que é esperado para a maioria das espécies de anuros (DUELLMAN; TRUEB, 1994). O período de maior atividade ocorreu na primeira metade da noite, semelhante ao registrado para os turnos de vocalização dos anuros em Poços de Caldas, em Minas Gerais (CARDOSO; MARTINS, 1987; CARDOSO; HADDAD, 1992). De maneira geral, outros estudos de atividade de vocalização de anuros mostraram que a concentração de cantos na primeira metade da noite pode estar relacionada com a redução do gasto energético e com a queda da temperatura do ar na segunda metade da noite (e.g. CARDOSO; MARTINS, 1987; NARINS et al., 2007). Por outro lado, nós observamos que muitas espécies permaneceram em atividade de vocalização durante a madrugada (e.g. P. nanus, H. bischoffi e $P$. distincta), indicando que outros fatores podem atuar no controle do gasto energético durante a vocalização.

A duração da atividade de vocalização dos anuros varia entre indivíduos e entre espécies, resultado de interações de níveis hormonais (andrógenos e corticosteroides), características do canto de anúncio e comportamento dos machos (EMERSON, 2001; ZINA; HADDAD, 2005). Espécies que possuem uma baixa taxa de vocalização e intensidade do canto podem vocalizar por mais tempo durante a noite (e.g. H. bischoffi- 
como Hyla multilineata em HEYER et al., 1990) do que espécies que possuem uma alta taxa de repetição e intensidade do canto (e.g. D. minutus - como Hyla minuta em HEYER et al., 1990).

Com esse estudo nós registramos o primeiro inventário de anuros na área de Floresta Ombrófila Densa da Serra do Tabuleiro, preenchendo uma das lacunas de conhecimento para Santa Catarina, com registro de três espécies relevantes para a conservação e com informações ecológicas da comunidade local, o que reforça a importância da região como área prioritária para a conservação da Mata Atlântica.

\section{Agradecimentos}

Agradecemos ao Hotel Plaza Caldas da Imperatriz e ao F. M. Brüggemann pelo apoio e suporte logístico deste trabalho. M. Wachlevski e L. K. Erdtmann receberam bolsa de iniciação científica do Hotel Plaza Caldas da Imperatriz/Universidade Federal de Santa Catarina. P.C.A. Garcia agradece pela bolsa de produtividade a pesquisa concedida pelo CNPq. Os espécimes de anuros foram coletados sob a licença número 141/02 (IBAMA).

\section{Referências}

CANAVERO, A.; ARIM, M.; NAYA, D. E.; CAMARGO, A.; ROSA, I.; MANEYRO, R. Calling activity patterns in an anuran assemblage: the role of seasonal trends and weather determinants. North Western Journal of Zoology, Oradea, v. 4, n. 1, p. 29-41, 2008

CARDOSO, A. J.; HADDAD, C. F. B. Diversidade e turno de vocalizações de anuros em comunidade neotropical. Acta Zoologica Lilloana, Tucumán, v. 41, p. 93-105, 1992.

CARDOSO, A. J.; MARTINS, J. E. Diversidade de anuros durante o turno de vocalizações em comunidade neotropical. Papéis Avulsos de Zoologia, São Paulo, v. 36, v. 23, p. 279-285, 1987.

COLWELL, R. K. EstimateS: Statistical estimation of species richness and shared species from samples. Version 8.2. 2009. Disponível em: <pur.oclc.org/estimates>.

CONTE, C. E.; MACHADO, R. A. Riqueza de espécies e distribuição espacial e temporal em comunidade de anuros (Amphibia, Anura) em uma localidade de Tijucas do Sul, Paraná, Brasil. Revista Brasileira de Zoologia, Curitiba, v. 22, n. 4, p. $940-$ 948, 2005.

CRUMP, M. L.; SCOTT JR, N. J. Visual encounter surveys. In: HEYER, W. R.; DONNELLY, M. A.; MCDIARMID, R. W.; HAYEK, L. C.; FOSTER, M. S. (Ed.). Measuring and monitoring biological diversity. Standard methods for amhibians. 1. ed.
Washington and London: Smithsonian Institution Press, 1994. p. 84-91.

DUELLMAN, W. E.; TRUEB, L. Biology of amphibians. Baltimore and London: The Johns Hopkins University Press, 1994. $670 \mathrm{p}$.

EMERSON, S. B. Male advertisement calls. Behavioral variation and physiological processes. In: RYAN, M. J. (Ed.). Anuran communication. Washington and London: Smithsonian Institution Press, 2001. p. 36-44.

FROST, D. Amphibian Species of the World. Version 5.6 (9 de janeiro de 2013). 2013. Eletronic Database. Disponível em: $<$ http:// research.amnh.org/herpetology/amphibia/index.html>.

FUNDAÇÃO SOS MATAATLÂNTICA. Atlas dos remanescentes florestais da Mata Atlântica. Período 2008-2010. São Paulo: Fundação SOS Mata Atlântica/Instituto Nacional de Pesquisas Espaciais, 2010. 60 p.

GARCIA, P. C. A.; VINCIPROVA, G. Range extensions of some anuran species for Santa Catarina and Rio Grande do Sul States, Brazil. Herpetological Review, St. Louis, v. 29, n. 2, p. 117-118, 1998.

GARCIA, P. C. A.; CARAMASCHI, U.; KWET, A. O status taxonômico de Hyla cochranae Mertens e recaracterização de Aplastodiscus A. Lutz (Anura, Hylidae). Revista Brasileira de Zoologia, Curitiba, v. 18, n. 4, p. 1197-1218, 2001.

GARCIA, P. C. A.; PEIXOTO, O. L.; HADDAD, C. F. B. A new species of Hypsiboas (Anura: Hylidae) from the Atlantic Forest of Santa Catarina, Southern Brazil, with comments on its conservation status. South American Journal of Herpetology, São Paulo, v. 3, n. 1, p. 27-35, 2008.

HADDAD, C. F. B.; TOLEDO, L. F.; PRADO, C. P. A.; LOEBMANN, D.; GASPARINI, J, L.; SAZIMA, I. Guia de anfíbios da Mata Atlântica: diversidade de biologia. São Paulo: Anolisbooks, 2013. 544 p.

HEYER, W. R. Taxonomic and natural history notes on frogs of the genus Centrolenella (Amphibia: Centrolenidae) from Southeastern Brazil and adjacent Argentina. Papéis Avulsos de Zoologia, São Paulo, v. 36, p. 1-21, 1985.

HEYER, W. R.; RAND, A. S.; CRUZ, C. A. G.; PEIXOTO, O. L.; NELSON, C. E. Frogs of Boracéia. Arquivos de Zoologia, São Paulo, v. 31, n. 4, p. 237-410, 1990.

KLEIN, R. M. Fitofisionomia, importância e recursos da vegetação do Parque Estadual da Serra do Tabuleiro. Sellowia, Itajaí, v. 33, p. 5-54, 1981.

KOVACH, W. L. Oriana for Windows, version 2.02. Pentraeth: Kovach Computer Services, 2004.

KUNZ, T. S.; GHIZONI JR, I. R.; SANTOS, W. L.A.; HARTMANN, P. A. Nota sobre a coleção herpetológica da Universidade Federal de Santa Catarina (UFSC). Biotemas, Florianópolis, v. 20, n. 3, p. 127-132, 2007.

KWET, A.; LInGNAU, R.; DI-BERnARDO, M. Pró-Mata. Anfíbios da Serra Gaúcha, sul do Brasil. 2. ed. Porto Alegre: EDPUCRS, 2010. 108 p.

LUCAS, E. G. Diversidade e conservação de anfíbios anuros no estado de Santa Catarina, Sul do Brasil. 2008. 202 f. Tese (Doutorado em Ciências) - Universidade de São Paulo, São Paulo. 2008 . 
LUCAS, E. M.; GARCIA, P. C. A. Amphibia, Anura, Hylidae Rafinesque, 1815 and Hylodidae Günther, 1858: Distribution extension and new records for Santa Catarina, southern Brazil. Check List, Rio Claro, v. 7, n. 1, p. 13-16, 2011.

MARCELINO, V. R.; HADDAD, C. F. B.; ALEXANDRINO, J. Geographical distribution and morphological variation of striped and nonstriped populations of the Brazilian Atlantic Forest treefrog Hypsiboas bischoffi (Anura: Hylidae). Journal of Herpetology, Salt Lake City, v. 43, n. 2, p. 351-361, 2009.

MMA-MINISTÉRIO DO MEIO AMBIENTE. Avaliação de ações prioritárias para a conservação da biodiversidade da Mata Atlântica e Campos Sulinos. Brasília: Conservation International do Brasil, Fundação SOS Mata Atlântica, Fundação Biodiversitas, Instituto de Pesquisas Ecológicas, Secretaria do Meio Ambiente do Estado de São Paulo, SEMAD/Instituto Estadual de Florestas-MG, 2000. 40 p.

NARINS, P. M.; FENG, A. S; FAY, R. R.; POPPER, A. N. Hearing and Sound Communication in Amphibians. New York: Springer, 2007. $366 \mathrm{p}$

NUNES, I.; KWET, A.; POMBAL JR., J. P. Taxonomic revision of the Scinax alter species complex (Anura: Hylidae). Copeia, Lawrence, n. 3, p. 554-569, 2012.

PEEL, M. C.; FINLAYSON, B. L.; MCMAHON, T. A. Updated world map of the Köppen-Geiger climate classification. Hydrology and Earth System Sciences, Delft, v. 11:1633-1644. Doi:10.5194/ hess-11-1633, 2007.

PRADO, C. P. A.; UETANABARO, M.; HADDAD, C. F. B. Breeding activity patterns, reproductive modes, and habitat use by anurans (Amphibia) in a seasonal environment in the Pantanal, Brazil. Amphibia-Reptilia, Leiden, v. 26, p. 211-221, 2005.
SANTOS, T. G.; KOPP, K.; SPIES, M. R.; TREVISAN, R.; CECHIN, S. Z. Distribuição temporal e espacial de anuros em uma área de Pampa, Santa Maria, RS. Iheringia, Série Zoologia, Porto Alegre, v. 98, n. 2, p. 244-253, 2008.

SCOTT JR, N. J.; WOODWARD, B. D. Surveys at breeding sites. In: HEYER, W. R.; DONNELLY, M. A.; MCDIARMID, R. W.; HAYEK, L. C.; FOSTER, M. S. (Ed.). Measuring and monitoring biological diversity. Standard methods for amhibians. 1. ed. Washington and London: Smithsonian Institution Press, 1994. p. 118-124.

SUBIRÁ, R. J.; SOUZA, E. C. F.; GUIDORIZZI, C. E.; ALMEIDA, M. P.; ALMEIDA, J. B.; MARTINS, D. S. Avaliação científica do risco de extinção da fauna brasileira - Resultados alcançados em 2012. Biodiversidade Brasileira, Brasília, v. 2, n. 2, p. 17-24, 2012.

WACHLEVSKI, M.; ROCHA, C. F. D. Amphibia, Anuran, resting of Baixada do Maciambu, municipality of Palhoça, state of Santa Catarina, Brazil. Check List, Rio Claro, v. 6, n. 4, p. 602-604, 2010.

ZAR, J. H. Biostatistical analysis. New Jersey: Prentice Hall, 1999. $663 \mathrm{p}$.

ZINA, J.; HADDAD, C. F. B. Reproductive activity and vocalizations of Leptodactylus labyrinthicus (Anura: Leptodactylidae) in southeastern Brazil. Biota Neotropica, Campinas, v. 5, n. 2, p. $1-11,2005$. 